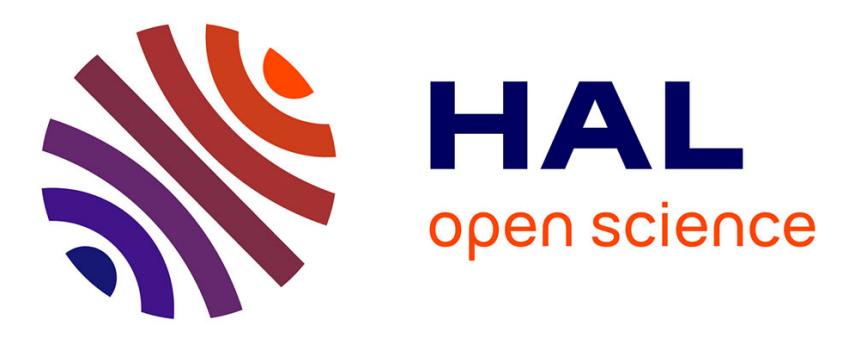

\title{
Analysis and specificities of adhesive forces between microscale and nanoscale.
}

Michaël Gauthier, Sébastien Alvo, Jérôme Dejeu, Brahim Tamadazte, Patrick Rougeot, Stéphane Régnier

\section{- To cite this version:}

Michaël Gauthier, Sébastien Alvo, Jérôme Dejeu, Brahim Tamadazte, Patrick Rougeot, et al.. Analysis and specificities of adhesive forces between microscale and nanoscale.. IEEE Transactions on Automation Science and Engineering, 2013, 3, pp.562-570. 10.1109/TASE.2013.2248150 . hal-00869504

\section{HAL Id: hal-00869504 https://hal.science/hal-00869504}

Submitted on 3 Oct 2013

HAL is a multi-disciplinary open access archive for the deposit and dissemination of scientific research documents, whether they are published or not. The documents may come from teaching and research institutions in France or abroad, or from public or private research centers.
L'archive ouverte pluridisciplinaire HAL, est destinée au dépôt et à la diffusion de documents scientifiques de niveau recherche, publiés ou non, émanant des établissements d'enseignement et de recherche français ou étrangers, des laboratoires publics ou privés. 


\title{
Analysis and specificities of adhesive forces between microscale and nanoscale
}

\author{
Michaël Gauthier*Member, IEEE, Sébastien Alvo ${ }^{\dagger *}$, Jérôme Dejeu ${ }^{\ddagger}$, Brahim Tamadazte*, Patrick Rougeot* and \\ Stéphane Régnier ${ }^{\dagger}$ Member, IEEE.
}

\begin{abstract}
Despite a large number of proofs of concept in nanotechnologies (e.g. nanosensors), nano electromechanical systems (NEMS) hardly come to the market. One of the bottlenecks is the packaging of NEMS which require handling, positioning, assembling and joining strategies in the mesoscale (from $100 \mathrm{~nm}$ to $10 \mu \mathrm{m}$, between nanoscale and microscale). It requires models of the interaction forces and adhesion forces dedicated to this particular scale. This paper presents several characteristics of the mesoscale in comparison with nanoscale and microscale. Firstly, it is shown that the distributions of charges observed on the micro-objects and meso-objects would have negligible effects on the nano-objects. Secondly, the impact of both chemical functionalisation and physical nanostructuration on adhesion are presented. Thirdly, the van der Waals forces are increased by local deformations on the mesoscale contrary to the nanoscale where the deformation is negligible. This article shows some typical characteristics of the mesoscale.
\end{abstract}

Note to Practitioners - Micro and nanorobotics covers a high range from nanometers to micrometers which represents six orders of magnitude. Most of the microassembly activities have been focused on micro-objects whose size is $10 \mu \mathrm{m}$ or more when nanohandling provides solutions mainly for nano-objects up to $100 \mathrm{~nm}$. The interest in the medium scale (mesoscale) has been growing recently. This article presents an analysis of the behavioral characteristic of the objects on this scale in comparison with the two others. It shows that specificities exist on the mesoscale and illustrates the requirement of original micro-nanorobotics at this particular scale.

Index Terms - Microscales, nanoscales, mesoscale, micronanohandling, modeling, interaction forces

\footnotetext{
*FEMTO-ST Institute, AS2M department, Universit de FrancheComt/CNRS/ENSMM/UTBM, 24 rue Savary, 25000 Besanon, France.

Email: michael.gauthier@femto-st.fr

$\dagger$ Institut des Systèmes Intelligents et Robotique (ISIR) Pyramide - T55/65, CC 173 - 4 Place Jussieu, 75005 Paris - France

Email: \{sebastien.alvo, stephane.regnier\}@isir.upmc.fr

†Département de Chimie Moléculaire (DCM), UMR CNRS 5250, Université Joseph Fourier, ICMG FR CNRS 2607, BP53, 38041 Grenoble Cedex 9 - France

Email: jerome.dejeu@ujf-grenoble.fr
}

\section{INTRODUCTION}

Micro and nanorobotics have provided a lot of handling, manipulation and assembly strategies on both micro and nanoscales during the last twenty years [1-21]. However, most of the micro-assembly activities have been focused on microobjects whose size is $10 \mu \mathrm{m}$ or more when nanohandling provides solutions mainly for nano-objects which are smaller than $100 \mathrm{~nm}$.

On the one hand, top-down approaches based on contact manipulation and tweezers are proposed [1], [2], [3], [4]. The main challenge is to tackle adhesion which becomes typically predominant around several hundred micrometers [5]. In order to avoid adhesion, authors have proposed to use self-assembly processes based on capillary forces [6], [7], [8], magnetic principle [9] or dielectrophoresis [10], [11]. These articles propose new ways to perform micro-assembly of complex and hybrid microsystems [3], [4].

On the other hand, nanomanipulation is mainly based on self-assembly using chemical processes [12], [13]. Authors have also proposed to manipulate nano-objects and typically Carbon NanoTube (CNT) or macromolecules with nanotweezers [14], [15], [16]. The objective is mainly to build nanocomponents (e.g. based on CNT) or to functionnalise surfaces (e.g. based on Self-Assembly Monolayer - SAM [17]).

On the mesoscale between $100 \mathrm{~nm}$ and $10 \mu \mathrm{m}$ some new exploratory works have been reported based on contact handling [18], [19] and based on non-contact manipulation [20], [21].

If we compare manufactured objects to biological ones, this mesoscale between nanoscale and microscale is the dimension of biological cells: the elementary components of life. In the biological domain, cells create a bridge between DNA on the nanoscale and tissues on the microscale (see figure 1). This multiscale assembly enables the construction of biological tissues with highly advanced properties e.g. self-reparable materials, powerful actuation. In comparison, the advent of micro-assembly strategies on the mesoscale could be a high challenge in order to build a bridge between microsystems and nanotechnologies. Fulfilling the lack of methods and technology on the mesoscale should open the way to smart and adaptive materials in several applications: energy harvesting, security and Structural Health Management (SHM).

The study of mesoscale assembly requires a better under- 


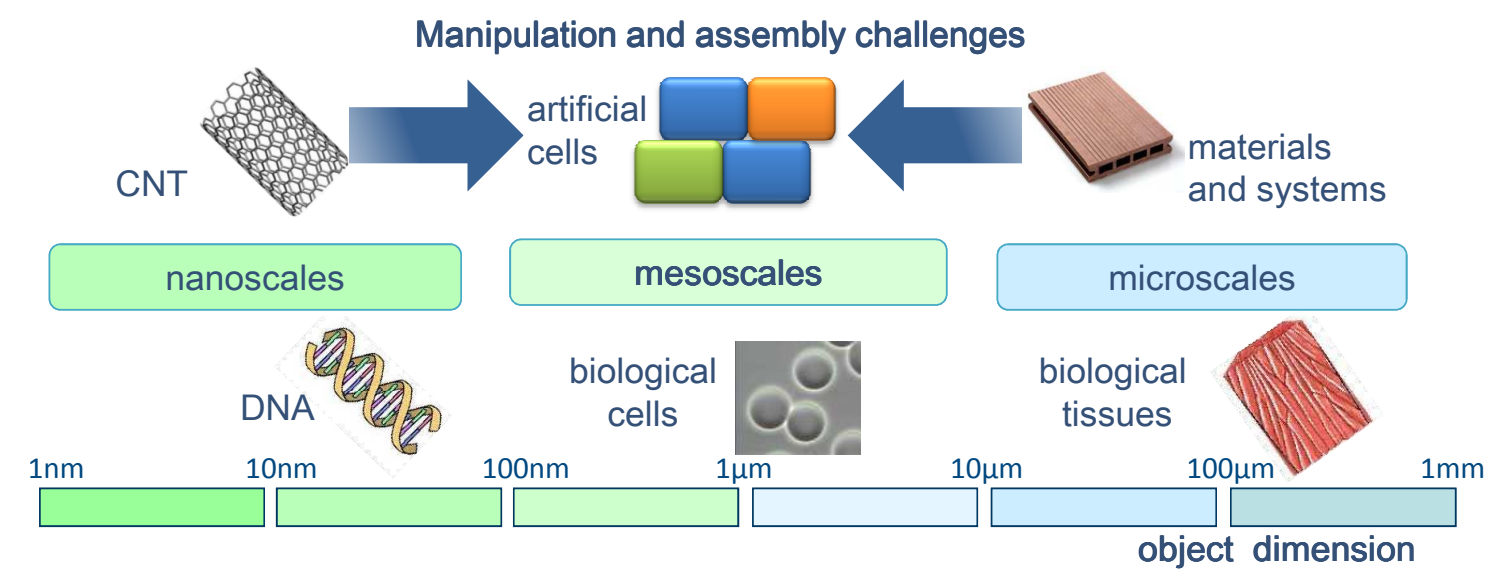

Fig. 1. Comparison between multiscaled biological world and manufactured products.

standing of object's behaviour in this scale which is significantly different to nanoscale and microscale. The objective of this paper is to present some characteristics in the behaviour of objects in mesoscale. It particularly focuses on the adhesion forces which are the most critical perturbations in contact micro-nanohandling (gripping, pushing). The next section emphasises the different current methods to model adhesion on the microscale and the nanoscale. The measurement of forces is essential to validate model and is presented in section III. Some typical significant coupling effects on the mesoscale are going to be presented in the following sections.

\section{Modeling THE MiCRO-NANOWORLDS}

Both ways to model adhesion are used in the literature: (i) based on nanoscale analysis, adhesion forces are built as the sum of the interaction forces (e.g. van der Waals forces); (ii) based on microscale analysis, where adhesion calculation is based on energetic models (e.g. JKR [22], DMT [23]). In both cases, the models propose analytic equations in the case of a contact between a sphere and a plane.

\section{A. From the microscale modeling...}

Top-down models are currently used to model adhesion. Energetic modeling based on continuum mechanics can be used to estimate the pull-off force between objects. The most famous models are the JKR [22] and the DMT [23] models which are limited to simple geometries. They are wildly used to estimate the force required to break the contact surface between objects: pull-off force. However, they provide a global value of the pull-off force without modeling the impact of each elementary effects (capillary force, electrostatic force, van der Waals force). This kind of model is not flexible: adding a complementary force or changing the geometry is complex. This is one of the major drawbacks of this approach.

\section{B. ... to the nanoscale modeling}

Adhesion is induced by several phenomena as van der Waals, electrostatic and capillary forces [24]. Each of them depends on several parameters (materials, roughness, humidity, temperature, etc.) and are due to nanophenomena.
Concerning van der Waals forces, it is commonly assumed to be additive forces. Consequently the total van der Waals forces between two objects $S_{1}$ and $S_{2}$ are the sum of forces applied on atoms of $S_{2}$ by atoms of $S_{1}$ [25], [26], [27]. Adhesion is currently modeled by considering a minimal distance $z=z_{0}$ between both objects. $z_{0}$ is usually set to $0.3 \mathrm{~nm}$ which is the equilibrium distance between two atoms in a vacuum. Throughout this paper, we consider interaction forces between a glass sphere of radius $r_{2}$ and a plane. The van der Waals forces induced by this geometry are [1]:

$$
F_{v d w}=-\frac{A_{12} r_{2}}{6 z^{2}}
$$

where $z=z_{0}$ for contacting objects and $A_{12}$ is the Hamaker coefficient $\left(A_{12}=6.5 \times 10^{-20} \mathrm{~J}\right.$ for glass-glass contact).

Principal electrostatic forces are due to surface charges added by friction or chemical treatments (e. g. cleaning, oxidization). The electrostatic force applied by a plane (surface charges $\sigma_{1}$ ) on a sphere (surface charges $\sigma_{2}$ ) is done by:

$$
\vec{F}_{e s}=2 \pi r_{2}^{2} \frac{\sigma_{1} \sigma_{2}}{\epsilon_{3}} \overrightarrow{n_{1}},
$$

where $\epsilon_{3}$ is the electrical permittivity of the environment, and $\overrightarrow{n_{1}}$ is the normal of the plane.

An electrostatic force appears also because of the equilibrium of the Fermi level when two conductive materials are placed in contact [28]. In this case, a potential difference $V_{F}$ (typ. from 0 to $0.5 \mathrm{~V}$ ) appears between both objects and the interaction force is calculated by:

$$
F_{V_{F}}=\frac{\pi \epsilon_{3} r_{2} V_{F}^{2}}{z_{F}}
$$

where $z_{F}$ is the interaction distance from which the tunneling effect starts which is typically $1 \mathrm{~nm}$ in the case of really smooth surfaces [28].

Capillary forces $F_{\text {capil }}$ are due to water meniscus created thanks to ambient humidity or adsorbed layers [29] are done by:

$$
F_{\text {capil }}=4 \pi r_{2} \gamma_{3} \cos \theta
$$


where $\gamma_{3}$ is the surface tension of water and $\theta$ is the contact angle between the liquid and the solids.

The models presented above can be used to model pull-off force on the microscale. Indeed, the most natural way to model pull-off force is to sum the elementary forces calculated when both objects are in contact.

The major interest of this approach is the fact that each phenomenon can be decoupled. This bottom-up model of pull-off force leads us to understand and compare the origins of the adhesion.

We are going to base our models dedicated to the mesoscale on this approach which enables to study different phenomenon separately.

\section{FORCE MEASUREMENTS}

Experimental force measurements are required to validate the proposed models. Our analysis is based on the force measurement performed by an Atomic Force Microscope (AFM) which is currently used to measure interactions between nanospheres (radius of the AFM tip) and substrates. In order to measure forces on the mesoscale, micrometer beads are fixed on AFM cantilevers (figure 2).

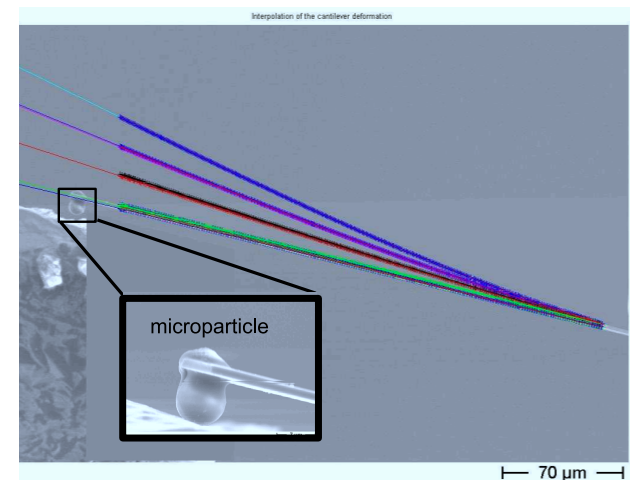

Fig. 2. Measurements of the cantilever deformations in SEM: each color represents a deformation of the beam which has been measured using vision algorithms.

The computation of measured forces on the mesoscale requires a careful study of the AFM where the force measurement is a function of the mechanical stiffness of the beam. From a mechanical point of view, the stiffness is directly linked to the mechanical boundary conditions at both ends of the beam. As the use of a micro-sphere in spite of the usual nanoscaled tip is able to significantly change the mechanical boundary conditions on the beam, we studied the mechanical behavior of the AFM beam in mesoscale measurements. To model the general behavior of a cantilever, we considered the deformation in the case of a clamped beam. The general boundary conditions include a force $F_{y}$ and a torque $T$ applied on the extremity of the beam. Considering a position $x$ along the beam, its deflection $y(x)$ classically verifies:

$$
y(x)=\frac{1}{E I}\left(-\frac{F_{y}}{6} \cdot x^{3}+\frac{L \cdot F_{y}-T}{2} \cdot x^{2}\right),
$$

where $E$ is Young's modulus, $L$ is the length of the beam, and $I$ is the momentum of inertia of the beam section. The stiffness of the beam can be expressed by:

$$
\frac{F_{y}}{y(L)}=\frac{3 E I}{L^{3}}\left(1-\frac{3}{2} \cdot \frac{T}{L \cdot F_{y}}\right)^{-1} .
$$

The ratio between $T$ and $L . F_{y}$ is driven by the boundary conditions at the extremity of the beam. For example, the clamped free condition is characterized by $\frac{T}{L \cdot F_{y}}=0$. The clampedclamped deformation is defined by $\frac{T}{L \cdot F_{y}}=0.5$. Between both cases, the stiffness is increased by a factor of 4 .

The identification of the boundary conditions can thus be found by measuring the whole deformation $y(x)$ and by identifying the ratio $\frac{T}{L \cdot F_{y}}$. The whole deformation $y(x)$ of the beam has been measured in a SEM and computed using (5) in order to define the ratio $\frac{T}{L \cdot F_{y}}$ (figure 3 ). We have shown that the ratio $\frac{T}{L \cdot F_{y}}$ is negligible compared to 1 (figure 3 ): the beam follows the deformation of a clamped-free beam. Consequently, current force measurement principles based on AFM, usually used on the nanoscale can be used on the mesoscale.

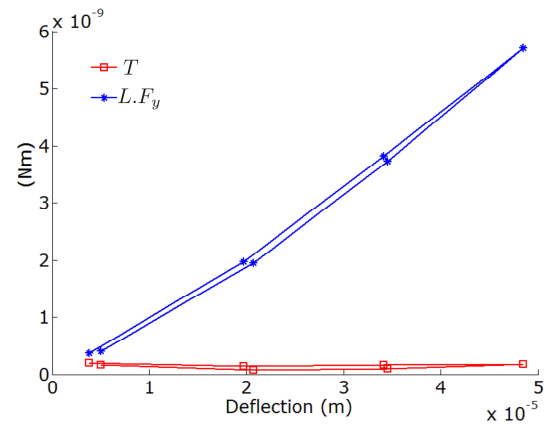

Fig. 3. Identified force momentum $F_{y} . L$ and torque $T$ at the end of the cantilever in function of the deflection: $T$ is negligible compared with $L . F_{y}$ which is a characteristic behavior of a clamped free beam.

\section{SCALE EFFECT ON ELECTROSTATIC FORCES}

The objective of this section is to present, in a concrete case, the scale effect of electrostatic forces compared to van der Waals forces.

On non-conductive materials, surface charges could appear with tribo-electrification and the level of charges is typically unpredictable. Indeed, no reliable models of triboelectrification currently exist in the state of the art. Consequently, in order to evaluate the order of magnitude of the electrostatic force $F_{e s}$, experiments have been done on a silicon plane and a borosilicate sphere. Typical surface charges about $\sigma=0.15 \mathrm{mCm}^{-2}$ have been determined using (2) during experiments. This value gives an example of charges which can be induced by tribo-electrification.

This electrostatic force $F_{e s}$ induced by tribo-electification can be compared with the electrostatic force $F_{V_{F}}$ induced by contact electrification. We are considering the maximal Fermi potential difference $V_{F}=0.5 \mathrm{~V}$ which maximizes the force $F_{V_{F}}$ [28]. 
Figure 4 represents the scale effect on the electrostatic force induced by tribo-electrification, the maximal electrostatic force induced by Fermi equilibrium and the van der Waals forces for a glass sphere. The first result deals with the Fermi equilibrium which induces a negligible force compared with the others. Secondly, the van der Waals forces are proportional to the radius of the bead (1), whereas the electrostatic force $F_{e s}$ is proportional to its square (2). This difference induces different scale effects on both phenomena. In the case of glass, it can be seen in figure 4 that the influence of the electrostatic force induced by tribo-electrification has to be considered for objects whose size is greater than $1 \mu \mathrm{m}$. On the nanoscale, the electrostatic forces become negligible compared to van der Waals forces (see figure 5).

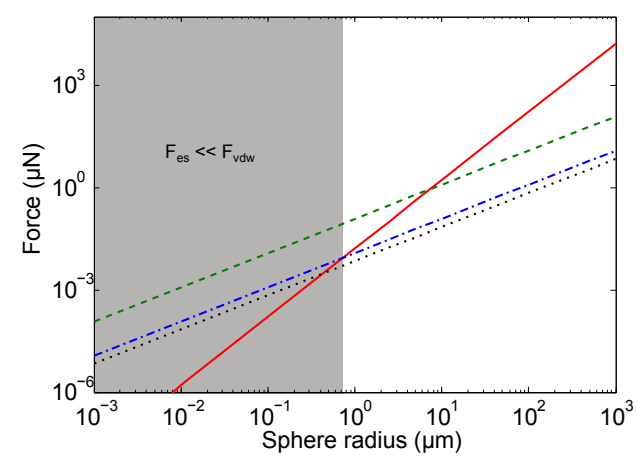

Fig. 4. Van der Waals (green dashed line) force, electrostatic force $F_{e s}$ induced by tribo-electrification (red solid line) and maximal electrostatic force $F_{V_{F}}$ induced by contact electrification (black dashed line) calculated between a sphere and a plane according to the sphere radius. The electrostatic force $F_{V_{F}}$ induced by contact electrification appears negligible. The electrostatic force $F_{e s}$ is considered as negligible as it is lower than the tenth of the van der Waals forces (blue dash-dot line). Considered materials for van der Waals and $F_{\text {es }}$ calculations are borosilicate glass, with $A_{12}=6.5 \times 10^{-20} \mathrm{~J}$, $z_{0}=0.3 \mathrm{~nm}$ and $\sigma=0.15 \mathrm{mC} . \mathrm{m}^{-2}$.

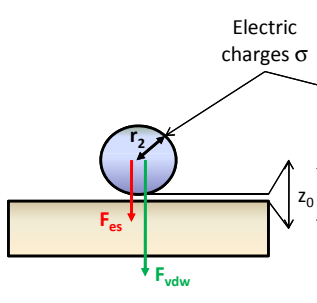

(a) Nanoworld

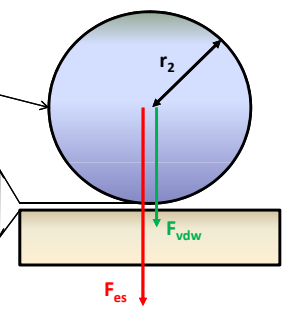

(b) Microworld

Fig. 5. Scale effect on electrostatic forces. On the nanoscale, electrostatic forces become negligible in front of van der Waals forces. On the microscale, electrostatic forces could become strong enough to lead adhesion phenomena.

The limit of $1 \mu \mathrm{m}$ is directly linked to the measured charge density used during our calculation. However, this result reports a global trend which shows that tribo-electrification can induce a significant force on the microscale whereas on the nanoscale it can be neglected. The mesoscale is characterized by the transition between both cases where both forces should be considered. In the case of non-conductive materials, the charge density can not be controlled and is unpredictable. Consequently, on the microscale and on mesoscale, the adhesion is difficult to predict and control for non-conductive microobjects without controlling the electrical charge density. On the nanoscale, the adhesion is mainly induced by the van der Waals forces which are repeatable.

\section{ChemiCAl FUnCtionalisation AND ELECTROSTATIC FORCES}

Based on the fact that electrostatic forces can have a predominant effect on the mesoscale, electrochemical effects can be exploited in order to control electrostatic surface charge density via chemical equilibrium between the surface and the liquid medium. Surface functionalisation of both objects and grippers can be obtained by different methods (physisorption, grafting, etc.).

The protonation of chemical functionnalisation have been widely studied on nano-objects [30], [31], [32]. On nanoscale, the electrostatic force is characterized by an interaction range of several tens of nanometers. On mesoscale, the impact of electrostatic forces induced by surface functionnalisations on beads has been observed experimentally in [33]. It shows the ability to obtain long interaction ranges (up to $100 \mu \mathrm{m}$ ), but the experiments have not been compared with a model. At least, electrostatic forces induced by an external voltage between mesoscaled objects have been modelled in [34].

We have proposed a model of electrostatic forces induced by chemical functionnalisation. Two chemical functionalisations have been tested: (i) the silane, 3 (ethoxydimethylsilyl) propyl amine (APTES); (ii) the silane, (3 aminopropyl) triethoxysilane (APDMES). Both chemical compounds (APTES, APDMES) used for surface functionalisation are amine functions $\mathrm{NH}_{2}$ which can be protonated or ionised to $\mathrm{NH}_{3}^{+}$ according to the $\mathrm{pH}$. In acidic $\mathrm{pH}$, the anime is totally ionised, then the ionisation decreases and is null in basic $\mathrm{pH}$ (between pH 9 and 12).

Our principle is also based on the protonation of silica, which enables the switch from $\mathrm{SiO}_{2}$ to $\mathrm{SiO}^{-}$according to the $\mathrm{pH}$. The combination of both effects enables to obtain a surface whose electrostatic charges switch from a positive value for low $\mathrm{pH}$, to a negative value for high $\mathrm{pH}$. These charges induce an interaction force $F_{e s}$ which could induces controllable adhesion phenomena.

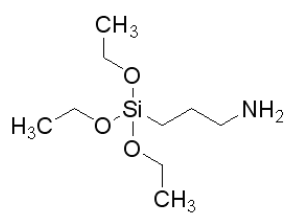

(a) APTES

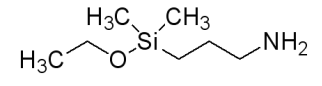

(b) APDMES
Fig. 6. Molecules used for the silica functionalisation.

Some force measurements were done on a functionalised plane using a cantilever with a non-funtionalised sphere. The results of the measurement are presented in figure 7. The $\mathrm{pH}$ significantly influences the forces between the cantilever and the surface. At a natural $\mathrm{pH}$, the electrostatic force is attractive. Indeed a pull-in force is measured when the bead comes to the 
surface (near $-60 \mathrm{nN}$ ) and a significant pull-off force is also measured $(-350 \mathrm{nN}$, figure $7(\mathrm{~b}))$. When the $\mathrm{pH}$ increases the electrostatic force becomes repulsive. The maximal repulsive forces reach $280 \mathrm{nN}$ and $770 \mathrm{nN}$ at $\mathrm{pH} 9$ and 12 respectively; and the adhesion forces disappear.

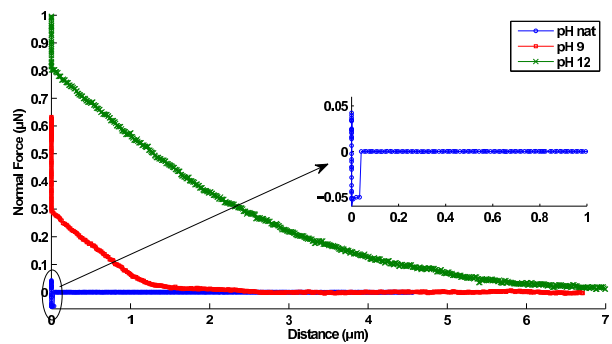

(a) Approach measurement

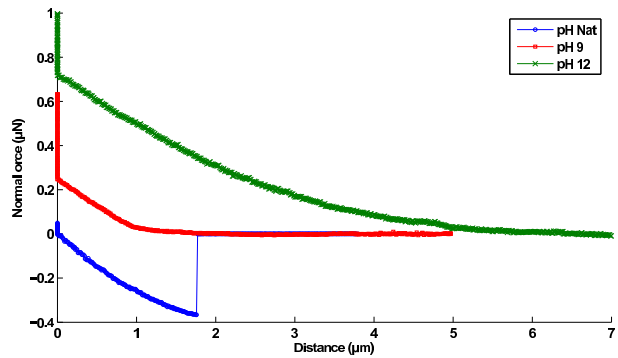

(b) Retract measurement

Fig. 7. Force-distance curve for the APTES functionalised substrate in wet medium at different levels of $\mathrm{pH}$ obtained with a tip whose spring constant is $0.3 \mathrm{~N} / \mathrm{m}$.

Moreover the interaction distance is typically several micrometers in mesoscale when the chemical electrostatic interaction force is only limited to tens of nanometers on the nanoscale [30], [31], [32]. Because of the size of the object, the interaction force on the mesoscale is significantly greater than on the nanoscale. It opens new methods in micro-nanorobotics: non-contact manipulation of mesoscaled objects in an electric field using surface charges controlled by chemistry can be considered.

The forces induced by chemical functionnalisations have different properties in each scale. Indeed, on nanoscale it can be considered as a short range force whose interaction distance is smaller than several nanometers. On mesoscale it is a long range force able to induce interaction up to several tens of micrometer. On microscale, weight is becoming greater than chemical based force (sedimentation) and this effect cannot be exploited

\section{IMPACT OF ROUGHNESS ON VAN DER WAALS FORCES}

Another important parameter which significantly modifies the adhesion is roughness. The impact of the roughness on van der Waals forces have been firstly modeled by [35] on simple rough profiles. More recently, models which consider roughness as a repartition of nanospheres have been proposed by [36], [37]. Some more complex models are also proposed in [38], [39]. These models are difficult to validate experimentally, because roughness is usually a random phenomenon.

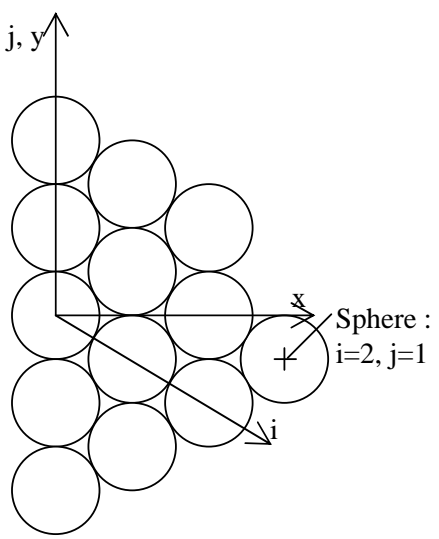

Fig. 8. Arrangement of the polystyrene $(P S)$ spheres on the substrate.

We have proposed to use nanostructurations in order to control roughness on surfaces during the force measurement. It enables the impact of roughness on interaction forces to be studied properly, and also enables the roughness in an application case to be controlled.

Let us consider the nanostructure described in figure 8 which represents the position of self-assembled polystryrene $(P S)$ nanospheres on a surface. These nanostructurations were built by the EMPA institute, Thun, Switzerland [40], [41] using self-assembly methods. In an application case and also during force measurements, the location of the sphere on the cantilever up to the structured surface cannot be controlled precisely. The bead on the cantilever $r_{2}$ touches the nanospheres $r_{1}$ on a non-controlled position. We have shown that the van der Waals force is included between a minimum and a maximum which verify [40]:

$$
\begin{gathered}
F_{\text {min }}=\frac{A_{12} r_{1} r_{2}}{r_{1}+r_{2}} \sum_{i, j}^{\mathbb{Z}^{2}} \frac{r_{2}+z_{0}+r_{1}}{6 l_{i j}\left(l_{i j}-r_{1}-r_{2}\right)^{2}} \\
F_{\text {max }}=\frac{A_{12} r_{1} r_{2}}{r_{1}+r_{2}} \sum_{i, j}^{\mathbb{Z}^{2}} \frac{\sqrt{\left(r_{2}+z_{0}+r_{1}\right)^{2}-\left(4 / 3 . r_{1}^{2}\right)}}{6 L_{i j}\left(L_{i j}-r_{1}-r_{2}\right)^{2}}
\end{gathered}
$$

where:

$$
\begin{aligned}
l_{i j}^{2} & =\left(r_{2}+z_{0}+r_{1}\right)^{2}+4 r_{1}^{2}\left(j^{2}-i j+i^{2}\right) \\
L_{i j}^{2} & =\left(r_{2}+z_{0}+r_{1}\right)^{2}+4 r_{1}^{2}\left(j^{2}-i j-j+i^{2}\right) .
\end{aligned}
$$

The comparison between values predicted by the model and the measurement, plotted in figure 9, shows a promising concordance. Moreover it shows a minimum of the interaction force which represents an optimum of adhesion reduction. Indeed, in the right-hand part of the figure (radius greater than $100 \mathrm{~nm}$ ), in the sums (7) and (8), 1 and 3 nanospheres induce significant forces respectively. In this case, the surrounding nanospheres are too far from the sphere $r_{2}$ and induce negligible forces. In the left-hand part of the figure (radius smaller than $100 \mathrm{~nm}$ ), the density of nanospheres is higher and the sum (7) and (8) include a lot of nanospheres. In this case, the smaller the nanospheres are, the higher the number of spheres in the sum is, the higher the total force is. In our experimental case, the optimal radius $r_{1}$ in order to minimize 


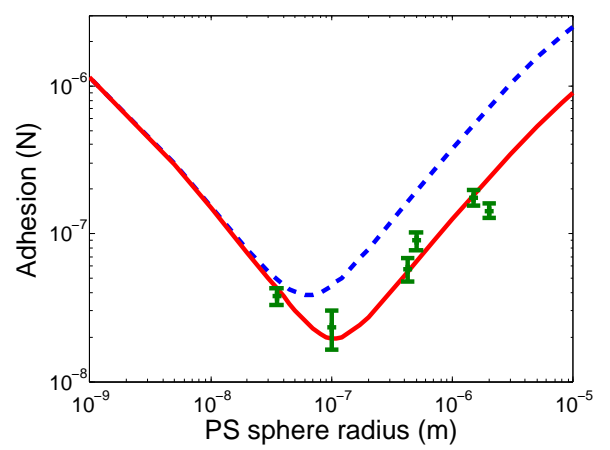

Fig. 9. Comparison between the model (minimal force, red solid line, and maximal force, blue dash line) and experimental measurements (error bar) on the structuring surface for borosilicate $20 \mu \mathrm{m}$ in function of the nanostructuration $r_{1}$.

the adhesion is between 45 and $100 \mathrm{~nm}$. This optimal value depends on the radius $r_{2}$ and the nature of the sphere placed on the cantilever.

The proposed model can be used to determine the diameter of the optimal PS nanospheres to be placed on a gripper to minimize adhesion force with a grasped sphere. Moreover, using (7) and (8), the model can be used for different types of materials knowing its Hamaker constant and for different geometry $r_{1}$ and $r_{2}$. Some first tests on nanostructured silicon grippers [43] have been presented in [40] (see in figure 10).

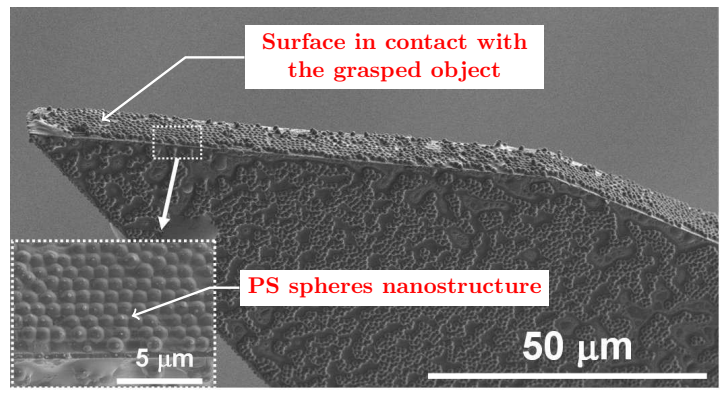

Fig. 10. Structured gripper by $P S$ particles of $1 \mu m$ : Joint work between EMPA institute, Thun, Switzerland, and FEMTO-ST institute, Besançon, France [40].

The optimal radius $r_{1}$ which represents the frontier between both asymptotic behaviors is around the frontier (typ. 100 $\mathrm{nm}$ ) between the nanoscale and the mesoscale. This example also shows behavioral differences between different scales and some specificities of the mesoscale compared to the nanoscale. Indeed, on the nanoscale the increase of the sphere radius $r_{2}$ induces a reduction of the force whereas on the mesoscale and microscale the increase of the sphere radius $r_{2}$ induces an increase of the force.

\section{INFLUENCE OF DEFORMATIONS ON VAN DER WAALS FORCES}

Van der Waals forces (1) are usually computed on nondeformed objects but local deformations are able to significantly increase their value on the mesoscale. Indeed, the greater part of van der Waals forces is due to the interaction of atoms near to the contact area. Therefore, deformations should be taken into account for the force calculation. This section shows that the coupling between van der Waals forces and deformations is also a characteristic of the mesoscale.

\section{A. Coupling principle}

Because of its short interaction range, the van der Waals forces are a surface force. In this model, we chose to replace this local force by an equivalent external load which induces a deformation. This deformation increases the contact surface and then the global van der Waals forces too. This coupled problem can be seen as an algorithm that sequentially uses two models (figure 11). The first one computes van der Waals forces according to the object shape. The other one computes deformation shape according to an external load. An iterative calculation is able to converge to the physical equilibrium.

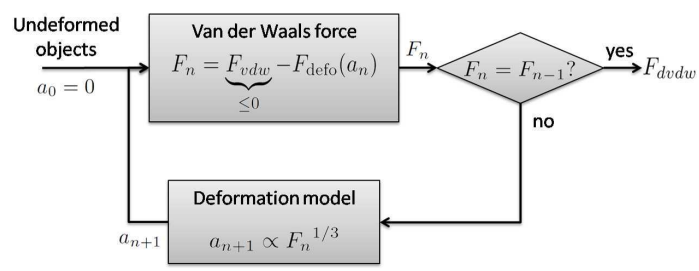

Fig. 11. Algorithm proposed for calculating the adhesion force $F_{d v d w}$ between two objects using the coupling between deformation and van der Waals forces. The algorithm starts with non-deformed objects so the initial contact radius $a_{0}$ is set to zero. The van der Waals forces $F_{n}$ can be computed as the sum of a deformable dependent part $F_{\text {defo }}$ and a non deformable one $F_{v d w}$ [44]. The deformation model based on the Hertz theory is able to provide the radius of the contact surface $a_{n+1}$ induced by $F_{n}$.

The coupling between van der Waals force and the local deformation can be illustrated on a contact between a sphere and a plane. The local deformation can be calculated by the Hertz defomation model, which enables the calculation of the radius $a$ of the contact surface in function of an external force. In order to calculate the van der Waals force on the deformed sphere, we consider a simplified geometry. Indeed, we assume that the deformed sphere is a truncated sphere where the radius of the removed spherical hat is $a$. It is then possible to calculate the van der Waals forces between the truncated sphere and the plane according to $a$ (details of the calculation can be found in [44]).

\section{B. Results}

The force $F_{d v d w}$ calculated using this modeling principle is presented in figure 12 in the case of silicon objects. A critical radius can be calculated from the model equations [44]:

$$
R_{c}=\frac{2 E^{2} z_{0}^{7}}{\left(1-\nu^{2}\right)^{2} A_{12}^{2}},
$$


where $E$ and $\nu$ are the mechanical characteristics of the objects. In the case of glass objects, $R_{c}=0.5 \mu \mathrm{m}$. If the sphere radius is smaller than this critical radius $R_{c}$, the computed force tends to be the classical van der Waals forces presented in eq. (1). So, on the nanoscale, the influence of deformation on van der Waals forces becomes negligible.

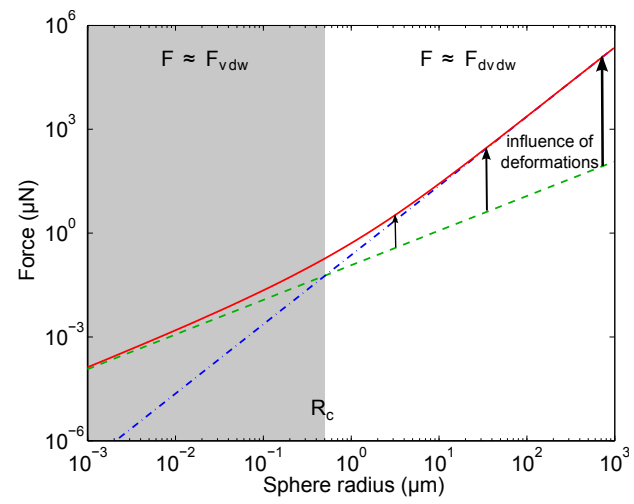

Fig. 12. Comparison of forces computed with classical and deformable van der Waals theories on the nanoscale and the microscale. The numerical solution of the deformable van der Waals (dvdw) model (red solid line) matches classical van der Waals (eq. (1), green dashed line) on nanoscale and analytical formula (eq. (12), blue dash-dotted line) on microscale.

The influence of deformations on the van der Waals force has only to be considered on the meso and micro-scales. We have shown in [44] that on the mesoscale, for a sphere radius $r_{2} \gg R_{c}$ the adhesion force tends to be:

$$
F_{d v d w}=-\frac{A_{12}^{3} r_{2}^{2}}{48 z_{0}^{9} E^{* 2}} .
$$

The difference between both force models increases with the radius of the sphere.

The coupling between the local deformation and the van der Waals force is a function of the scale considered (figure 13). Indeed, on the nanoscale, the coupling is negligible and the van der Waals force is proportional to the radius $r_{2}$ of the sphere (1). On the microscale, the coupling is significant and the van der Waals force on the deformed geometry is proportional to the square of the sphere radius $r_{2}$ (see asymptotic behavior on figure 13 and equation (12)). Mesoscale is the transition between both asymptotic cases where the coupling should be determined with numeric calculations.

\section{DISCUSSIONS}

This analysis illustrates the wide range from $1 \mathrm{~nm}$ to $1 \mathrm{~mm}$ of micro-nano-scales which includes 6 orders of magnitude with high differences. Each order of magnitude has its own characteristics and consequently its own scientific problematics. In order to differentiate each of them, we propose to use additional terms before micro and nano prefixes (see in figure 14). On the microscales, three different levels can be defined: iso-microworld (up to $10 \mu \mathrm{m}$ ), deca-microworld (for the medium scale) and hecto-microworld (down to $100 \mu \mathrm{m}$ ). These notations could be a solution to clearly classify scientific and technological works in function of their scale of validity.

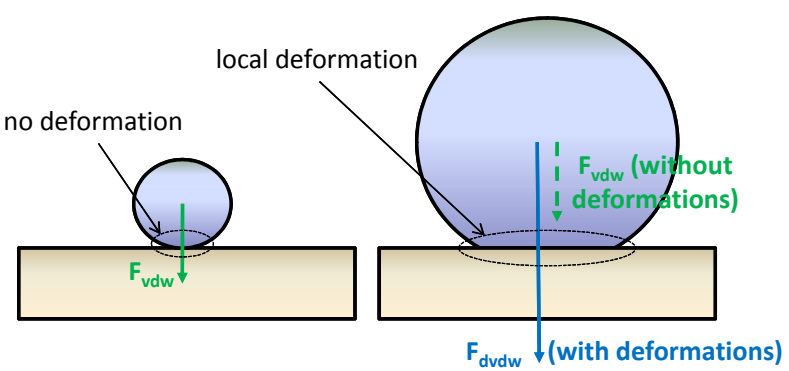
(a) Nanoworld
(b) Microworld

Fig. 13. Impact of deformation on the van der Waals forces.

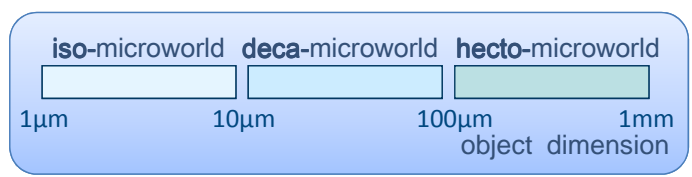

Fig. 14. Proposition of prefixes in order to differentiate scientific and technological results in function of the dimension of the object manipulated on the microscales: iso-microworld (up to $10 \mu \mathrm{m}$ ), deca-microworld (for the medium scale) and hecto-microworld (down to $100 \mu \mathrm{m}$ )

\section{CONCLUSION}

Manipulation and assembly on the micrometer scale (mesoscale) appear to be a high challenge for the future integration of nanotechnology in materials or systems. The micrometer seems to be a critical size characterized by couplings between several physical effects. Four features of physics on the mesoscale were highlighted in this paper: (i) electrostatic forces disturb micromanipulation whereas it seems to be negligible on the nanoscale, (ii) surface functionalisation enables interaction forces to be significantly modified, (iii) the impact of the roughness is different on the meso and the nanoscale and (iv) the mechanical deformations strongly increase the van der Waals force only on the microscale and the mesoscale. This paper illustrates the paradigm of the mesoscale placed between micro and nanoscales.

\section{ACKNOWLEDGMENT}

This work is supported by the european project FP7 FoF FAB2ASM (contract FoF-NMP-2010-260079) (http://fab2asm.eu/); by the Labex ACTION project (contract "ANR-11-LABX-01-01"); by the French RENATECH network and its FEMTO-ST technological facility and by the Equipex ROBOTEX project (contract "ANR-10-EQPX44-01"). We would also like to acknowledge the support of EMPA institut, Thun, Switzerland in nanostructuration.

\section{REFERENCES}

[1] M. Gauthier and S. Régnier, Robotic micro-assembly, IEEE Press, Wiley Edition, ISBN:9780470484173, 2010.

[2] N. Chaillet and S. Régnier, Microrobotics for Micromanipulation, Wiley Edition, ISBN: 978-1-84821-186-5, 2010.

[3] K. Rabenorosoa, C. Clévy, Q. Chen, and P. Lutz, Study of Forces during Micro-Assembly Tasks using Two-Sensing-Fingers Gripper, IEEE/ASME Transactions on Mechatronics, 99, 2011, 1-11, DOI (10.1109/TMECH.2011.2131673). 
[4] H. K. Chu, J. K Mills, and W. L. Cleghorn, Parallel microassembly with a robotic manipulation system, Journal of Micromechanics and Microengineering, 20(12), 2010, 125027.

[5] M. Gauthier, S. Régnier, P. Rougeot, and N. Chaillet, Forces analysis for micromanipulations in dry and liquid media, Journal of Micromechatronics, 3 (34), 2006, 389-413.

[6] C. Lenders, J.-B. Valsamis, M. Desaedeleer, A. Delchambre, and P. Lambert, "Assembly of a micro-ball-bearing using a capillary gripper and a microcomponent feeder", in Micro-assembly technologies and applications, IFIP Int. Fed. Inform. Process., vol. 260, pp. 265-75, 2008

[7] K. F. Bohringer, Self-assembly from nano to milli scales, Springer Ed., 2013.

[8] V. Sariola, M. Jääskeläinen, and Q. Zhou, Hybrid Microassembly Combining Robotics and Water Droplet Self-Alignment, IEEE Transactions on Robotics, 26 (6), 2010, 967-977.

[9] G. M. Whitesides and B. Grzybowski, Self-Assembly at All Scales, Science, 295 (5564), 2002, 2418-2421.

[10] M. Kharboutly, M. Gauthier, and N. Chaillet, Modeling the trajectory of a micro-particle in a dielectrophoresis device, Journal of Applied Physics, 106, 2009, 114312-318.

[11] M. Gauthier, E. Gibeau and D. Hériban, Submerged Robotic Micromanipulation and Dielectrophoretic Micro-object Release, in proc. of the IEEE ICARCV 2006 conference, Singapour, 6 pages, dec. 2006.

[12] A. Weddemann, I. Ennen, A. Regtmeier, C. Albon, A. Wolff, K. Eckstdt, N. Mill, M. K.-H. Peter, J. Mattay, C. Plattner, N. Sewald and A. Htten, I. Ennen et al. Review and outlook: from single nanoparticles to selfassembled monolayers and granular GMR sensors, Beilstein Journal of Nanotechnology, 1, 2010, 75-93.

[13] Y. Makoudi, C. Didiot, F. Palmino, E. Duverger, B. Kierren, D. Malterre , and F. Cherioux, Self-assembly of zwitterionic molecules on a $\mathrm{Au}(23$ 23 21) surface at low temperature, Surface Science 604, 2010, 27-31.

[14] Z. Wang, D. Li, J. Zhang, Z. Ji, and R. Qiu, Robotic nanoassembly: current developments and challenges, International Journal of Computer Applications in Technology, 41 (3-4), 2011, 185-194.

[15] S. Fatikow, V. Eichhorn, D. Jasper, M. Weigel-Jech, F. Niewiera, and F. Krohs, Automated nanorobotic handling of bio- and nanomaterials, 2010 IEEE Conference on Automation Science and Engineering (CASE), 6 pages, 2010.

[16] A. Bolopion, H. Xie, S. Haliyo and S. Rgnier, Haptic Teleoperation for 3D Microassembly of Spherical Objects. IEEE/ASME Transaction on Mechatronics, Vol 17, No 1, Pages 116-127, 2012

[17] J. Dejeu, M. Gauthier, P. Rougeot, and W. Boireau, Adhesion forces controlled by chemical self-assembly and $\mathrm{pH}$, application to robotic microhandling, ACS Applied Materials and Interfaces, 1 (9), 2009, 19661973.

[18] H. Xie and S. Régnier, Three-dimensional automated micromanipulation using a nanotip gripper with multi-feedback, J. Micromech. Microeng., Journal of Micromechanics and Microengineering, 19 (7), 2009, 7500917.

[19] R.T. Rajendra Kumar, S.U. Hassan, O. Sardan, V. Eichhorn, F. Krohs, S. Fatikow, and P. Boggild, Nanobits: customisable scanning probe tips, Nanotechnology, 20 (39), 2009, 395703

[20] K. Onda and F. Arai, Multi-beam bilateral teleoperation of holographic optical tweezers, Optics Express, 20 (4), 3633-3641, 2012.

[21] H. Maruyama, R. Litsuka, K. Onda, and F. Arai, Massive parallel assembly of microbeads for fabrication of microtools having spherical structure and powerful laser manipulation, in Proc. of the IEEE int. conf. in Robotics and Automation, 482-7, 2010.

[22] K. L. Johnson, K. Kendall, and A. D. Roberts, Surface energy and the contact of elastic solids, Proc. R. Soc. Lond. A, 324(1558), 1971, 301313.

[23] B. V. Derjaguin, V. M. Muller, and YU. P. Toporov, Effect of Contact Deformations on the Adhesion of Particles, Journal of Colloid and Interface Science, 53(2), 1975, 314-326.

[24] R. S. Fearing, Survey of Sticking Effects for Micro Parts Handling, in IEEE/RSJ Int. Conf. on Intelligent Robots and Systems, IROS, 95 (2), 1995, 212-217.

[25] H. C. Hamaker, The London - van der Waals attraction between spherical particules, Physica IV, 10, 1937, 1058-1070.

[26] J. N. Israelachvili, The Nature of van der Waals Forces, Contemporary Physics, 15(2), 1974, 159-177, 1974.

[27] V. A. Parsegian, Van der Waals forces. A Handbook for Biologists, Chemists, Engineers, and Physicists. Cambridge University Press, 2006, UK.

[28] M. Sausse-Lhernould, S. Rgnier and P. Lambert, Electrostatic forces in micromanipulations: review of analytical models and simulations including roughness. Applied Surface Science. Vol 253 Pages 62036210, 2010.

[29] P. Lambert, Capilary forces in Microassembly, Springer Ed, 2007.

[30] Jonathan A. Brant and Amy E. Childress. Membranecolloid interactions: Comparison of extended dlvo predictions with afm force measurements. Environmental Engineering Science, 19(6), 2002.

[31] Hasan Guleryuz, Arne K. Royset, Ingeborg Kaus, Claudine Filiatre, and Mari-Ann Einarsrud. Afm measurements of forces between silica surfaces. Chemistry and Materials Science Journal of Sol-Gel Science and Technolog, 62(3):460-469, 2012

[32] Jialin Wang and Roe-Hoan Yoon. Afm forces measured between gold surfaces coated with self-assembled monolayers of 1-hexadecanethiol. Langmuir, 24(15):78897896, 2008

[33] D.T. Nhan and G.H. Pollack. Effect of particle diameter on exclusionzone size. Int J Des Nat Ecodyn, 6(2):139144, 2011.

[34] M. Sausse Lhernould, P. Berke, T. J. Massart, S. Régnier, and P. Lambert. Variation of the electrostatic adhesion force on a rough surface due to the deformation of roughness asperities during micromanipulation of a spherical rigid body. Journal of Adhesion Science and Technology, 23:13031325, 2009

[35] H. Rumpf. Particle Technology. Chapman and Hall, 1990.

[36] Y. I. Rabinovich, J. J. Adler, A. Ata, R. K. Singh et B. M. Moudgil Adhesion between Nanoscale Rough Surfaces. I. Role of Asperity Geometry.. Journal of Colloid and Interface Science, 232:1016, 2000.

[37] E. R. Beach, G. W. Tormoen, R. Han, and J. Drelich. Pull-off force measurements between rough surfaces by atomic force microscopy. Journal of Colloid and Interface Science, 247(1):8499, 2002.

[38] M. Savia and Q. Zhou. Van der waals force computation of freely oriented rough surfaces for micromanipulation purposes. In IEEE/RSJ International Conference on Intelligent Robots and Systems, IROS10, page 56225627, 2010 .

[39] M. Savia, Q. Zhou, and H. N. Koivo. Simulating adhesion forces between arbitrarily shaped objects in micro/nano-handling operations. In proc. of the IEEE/RSJ IROS conf., pages 1722-27, 2004.

[40] J. Dejeu, M. Bechelany, L. Philippe, P. Rougeot, J. Michler, and M. Gauthier, Reducing the Adhesion between Surfaces Using Surface Structuring with PS Latex Particle, ACS Applied Materials \& Interfaces, 2(6), 2010, 1630-1636.

[41] J. Dejeu, M. Benchelany, P. Rougeot, L. Philippe, and M. Gauthier, Adhesion control for micro- and nano-manipulation, ACS NANO, 5(6), 2011, 4648-4657.

[42] J. Dejeu, M. Benchelany, E. Berodier, P. Rougeot, J. Michler, and M. Gauthier, Nanostructured nonadhesive surface for micro- and nanomanipulation, The journal of Physical Chemistry C, 116, 2012, 15117 15125 .

[43] J. Agnus, D. Hériban, M. Gauthier, and V. Pétrini, Silicon end-effectors for microgripping tasks, Precision Engineering, 33, 2009, 542-548.

[44] S. Alvo, P. Lambert, M. Gauthier, and S. Régnier, A van der Waals Force-Based Adhesion Model for Micromanipulation, Journal of Adhes. Sci. and Tech., 24(15-16), 2010, 2415-2428.

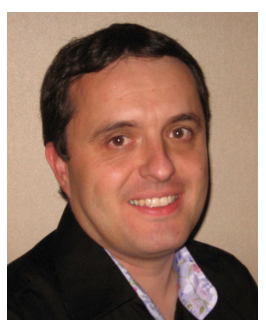

Michaël Gauthier received his $\mathrm{PhD}$ degree in $\mathrm{Au}-$ tomation and Robotics from the Universit de Franche Comté, France in 2002. He is currently CNRS Senior scientist research at FEMTO-ST institute and the head of the Automation and MicroMechatronics Systems (AS2M) Department. He has been granted by the French CNRS bronze medal (Early Career Award) in 2011. His research interests are focused on microworld modeling, micro and nano manipulation, dexterous microhandling and high speed control of non-contact micromanipulations. He is author or co-author of 28 journal papers and 42 conference papers in the field of micro-nano-robotics. 


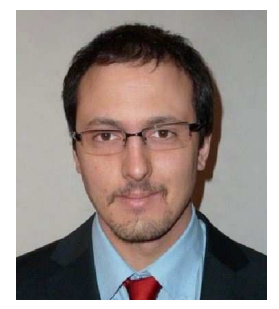

Sébastien Alvo received the M.S. degree and the $\mathrm{Ph} . \mathrm{D}$. degree in robotics and mechanical engineering from both University Pierre et Marie Currie, Paris, and University of Franche-Comté, Besanon, in 2008 and 2012, respectively. He is currently post doc at the Photonic and Nanostructures Laboratory, Marcoussis, France. His research interests are related to force sensing and modeling at the microscale and are currently focused on force measurements in SEM.

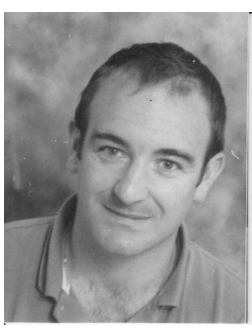

Jérôme Dejeu received the Ph.D. degree in physical chemistry from the University of Franche-Comtín 2007. He held a Research Scientist position at the FEMTO-ST Institute before joining the faculty of the University Joseph Fourier (Grenoble 1) where is Assistant Professor of Molecular Chemistry Department. His research interest includes the physicalchemistry phenomena at the interface as interaction, adsorption, and the comparison to the experimental measurements with the theory by the development of models.

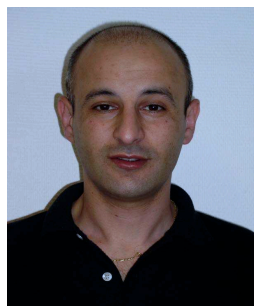

Brahim Tamadazte holds a Ph.D. in Automation and Computer Science from the "Universitde Franche-Comt" in 2009 and a MS degree in Robotics and Intelligent Systems from the "Université Pierre et Marie Curie (Paris VI)" in 2005, in France. Before, he has also trained as an engineer in $\mathrm{Au}$ tomation Systems at the Université Mouloud Mammeri de Tizi-Ouzou" from Algeria. During his Ph.D thesis, he has developed different vision algorithms and vision-based control laws (image-based visual servoing and pose-based visual servoing and direct visual servoing) for the automation of MEMS handling and assembly. After a post-doctoral position at the TIMC-IMAG Laboratory (GMCAO team, Grenoble, France), where he is involved in computer-assisted medical interventions (surgical robotics), he recruited as CNRS researcher at the FEMTOST Institute (AS2M department, Besanon, France) where he is involved in microrobotics surgery investigations.

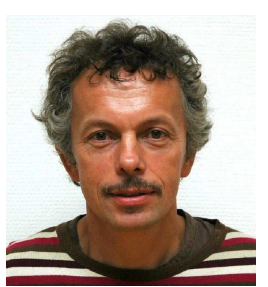

Patrick Rougeot is research engineer in the AS2M department of FEMTO-ST institute, France since 2000. He is working in the field of development and control of robotic equipment for 30 years. He works in the field of microrobotics and especially on Micro/Nanoforce measurement (SPECIMeN team). His objectives are developments and exploitations of measurement systems at micro- and nanoscopic scales. His research interests are focused on atomic force microscopes (AFM) for the characterization of the surface effects and on design and developments of tools for micromanipulations. The major activities of his research deals with the adhesion force measurement on structured and/or functionalized surfaces and polymer films.

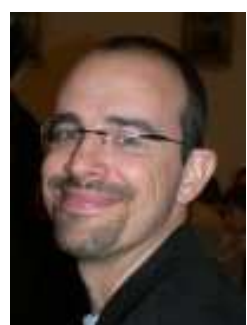

Stéphane Régnier received his $\mathrm{PhD}$ degree in Mechanics and Robotics from the University of Pierre and Marie Curie, Paris, France in 1996.

$\mathrm{He}$ is currently Professor at the Institute of Intelligent Systems and Robotics (ISIR), University of Pierre and Marie Curie, Paris, France. He is head of the micromanipulation team of ISIR since 2001. His research interests are focused to micro and nano manipulation, teleoperation and haptic feedback at the nanoscale, micromechatronics and biological cell characterization. 